Savina RAYNAUD

Université Catholique de Milan

\title{
DU CENTRE À LA PÉRIPHÉRIE : STRUCTURATION DES LANGUES EN DIACHRONIE, OU LA FORME INTERNE DES LANGUES ET SES AXES DE DÉVELOPPEMENT ${ }^{*}$
}

\section{De «qu'est-ce qu'une langue ? » à «qu'est-ce qu'une langue a ? ». Une connaissance comparative.}

Nous sommes si plongés dans le langage qu'il nous est difficile de répondre à des questions fondamentales le concernant.

Il est difficile de dire ce qu'est le langage, ce qu'est une langue. Il s'agit d'identifications qui nous engagent, de définitions ardues.

D'autres possibilités s'offrent à nous, moins ardues peut-être encore que non totalement évasives : en particulier, la possibilité de fonder une comparaison, ou bien de partir de l'identification de propriétés acquises, afin de parvenir à dire au moins ce que quelque chose a, si ce n'est ce que ce quelque chose est. Dans notre cas, ce que l'on peut attribuer aux langues, ce qui leur a été attribué, c'est d'être douées d'un centre et d'une périphérie.

Quoi d'autre est pourvu d'un centre et d'une périphérie ? En d'autres termes : à quoi allons-nous comparer les langues en leur attribuant un centre et une périphérie ? À une ville, plutôt qu'à une figure géométrique. À un système organique, comme le système cardio-vasculaire ou le système nerveux. D'autres auteurs enrichiront les termes de cette comparaison en lui ajoutant l'irradiation des cercles concentriques générés par la chute d'un objet dans l'eau, ou encore la formation progressive et symétrique des cristaux.

Quel est le poids qu'il convient d'attribuer à la connaissance qui avance par comparaisons? Il ne s'agit pas ici de comparaison entre des langues (en diachronie ou en synchronie), ni de linguistique historico-comparative ou de linguistique contrastive. La comparaison dont il est question s'instaure entre les faits linguistiques et d'autres faits, d'autres systèmes, d'autres œuvres de l'homme, d'autres organismes naturels. Quel est donc le poids qu'il faut attribuer à une telle comparaison? Il me semble que l'on ne peut pas sous-estimer l'importance de la comparaison en tant que processus de connaissance typiquement humain; d'une comparaison qui, même quand elle ne produit pas d'identités à tous les effets, suggère toutefois des ressemblances, des analogies, des proportions et, éventuellement, précise des différences, des inéquations, des disproportions.

Il s'agit par la suite de «gérer» comme il convient les termes de la comparaison. Le risque subsiste de sous-estimer les différences qui existent toutefois, de réduire l'écart entre le premier terme de la comparaison et le deuxième, de neutraliser les oppositions qu'un œil attentif ne manquerait pas de conserver.

\footnotetext{
* À mes collègues et amis Candido et Enrica Matasci ma reconnaissance la plus vive pour la traduction française de ces pages.
} 
J'essaierai ici de partir de l'observation des propriétés inhérentes aux « premiers » termes de la comparaison: la ville, in primis, mais pas seulement. Je vais partir des propriétés constituantes et de leur observation et description directe, avant de préciser les méthodes adoptées pour représenter, à l'échelle, de telles propriétés.

Afin de mener ces observations, $\mathrm{j}$ ' aurai recours à quelques auteurs, pragois et non pragois, de la première et de la deuxième génération du Cercle pour les premiers, leurs contemporains pour les autres, comme Wittgenstein, qu'ils précèdent de peu ou de beaucoup, comme Marty, et plus encore Humboldt, en remontant jusqu'au monde grec classique, et à Aristote.

\section{Un territoire habité à explorer, avant d'en établir la cartographie. La primauté de l'acte expressif-communicatif.}

La langue latine a deux manières distinctes de considérer la ville : urbs vs civitas.

«Urbs: quam cum locis manuque depicserit, ejusmodi conjunctionem tectorum oppidum, vel urbem appellarunt, delubris distinctam, spatiisque communibus ». ${ }^{1}$ "Civitas : civium multitudo in eodem loco habitans, eodemque jure vivens $»{ }^{2}$

Le Petit Prince, en visitant la sixième planète, met au point la différence entre les tâches du géographe et celles des explorateurs. ${ }^{3}$

Le cartographe doit attendre le reportage des explorateurs avant de pouvoir représenter un territoire avec la puissance d'une vision d'ensemble, en sauvegardant les proportions (l'uniformité de l'échelle adoptée) tout en intégrant sa représentation dans un système de repères (les points cardinaux) et en $\mathrm{y}$ introduisant un système de coordonnées (latitude et longitude).

En laissant de côté la métaphore, j'entends ici caractériser le travail métalinguistique (du phonologue, du grammairien, du lexicologue, du linguiste computationnel, du philosophe du langage), en le subordonnant à la collecte des données, à l'observation directe, à l'exploration et à l'analyse approfondie (du linguiste sur le terrain, du phonéticien, du lexicographe, du linguiste de corpus, du sociolinguiste). Et même s'il ne s'agit pas d'une relation de subordination, je l'envisage au moins dans une relation stable de coordination et d'implication mutuelle.

Sur la scène d'une si profonde exploration, la véritable protagoniste, la référence incontournable pour toute entreprise de représentation, c'est, comme

\footnotetext{
${ }^{1}$ Cic. in Fragmenta apud Nonium, c. 5 n. 27 - rep. ForCELLINI (1805).

${ }^{2}$ Cic. in Somnium Scipionis c. 3 - rep. ForCELLINI (1805).

Je remercie Tomáš Hoskovec d'avoir signalé que la même distinction existe en grec entre astu et polis et que, tandis qu'en latin le substantif abstrait civitas vient de civis, en grec polites est le dérivé du substantif polis. Il s'agit de deux processus inverses qui illustrent la complémentarité entre les parties et le tout, la dialectique entre appartenance et totalité, totalité qui est constituée et constitutive à la fois.

${ }^{3}$ Chap. XV.
} 
l'Ecole de Prague nous l'a appris, la communauté des parlants, incluant l'activité expressive et communicative de chaque locuteur.

\section{Une exploration systématique poursuivie dans le temps et ses cartographies}

La scène qui se déroule devant l'œil vigilant de l'explorateur est en effet un paysage en transformation dans le temps, dans l'espace, dans la communauté sociale, dans les formes du parler: le travail métalinguistique et son évaluation métathéorique ne peuvent faire abstraction, en ce qui concerne les langues historico-naturelles, d'une prise en charge de leurs variations diachroniques, diatopiques, des variations liées aux strates et aux dialectes.

Je suis de l'avis que l'enseignement de l'Ecole de Prague relatif à la permanence du système linguistique en tant que système dans l'évolution temporelle garde toute sa valeur et sa fécondité. Voilà pourquoi au lieu d'utiliser le terme «structures » j'aime mieux parler ici de «structurations », en laissant une fois de plus le devant de la scène aux communautés des parlants. Des structurations qui sont d'abord accomplies ${ }^{4}$ avant d'être décrites, repérées, documentées. Nous ne pouvons certes pas oublier le rôle parfois actif, de standardisation, normatif, de « disciplinement » exercé par les linguistes. C'est là le domaine de la politique linguistique et, plus en général, culturelle. La francophonie est concernée encore plus que d'autres aires linguistiques. Mais il s'agit, à mon avis, de rôles subordonnés au rôle fondamental rempli par le sujet de l'activité locutoire: la communauté qui hérite d'un idiome, qui en oriente dynamiquement les équilibres internes et extérieurs, qui en ratifie les lignes de force, abandonne toujours quelque chose et prend et transforme d'autres choses. Elle fait cela dans un régime d'échange communicatif mutuel et constant et dans un rapport incessant avec le monde qui l'entoure.

S'il existe un auteur, cher aux Pragois, qui s'est avancé à l'échelle planétaire, dans l'exploration de systèmes linguistiques nombreux et disparates, et qui a sérieusement médité sur la raison d'être de leur apparition, de leur devenir, de leur maturation et -parfois- de leur disparition, c'est bien Humboldt, un savant moderne de par sa pratique exceptionnelle du plurilinguisme, classique par sa familiarité avec le génie aristotélicien, avec le monde grec et latin, inébranlable dans sa façon de conjuguer sensibilité littéraire et curiosité à l'égard de la nature. L'Académie des Sciences de Berlin est encore de nos jours engagée dans l'édition posthume de son énorme travail de grammairien des langues exotiques. ${ }^{5}$

Il existe des pages de Humboldt qui aident, me semble-t-il, de façon extraordinaire à restituer à l'explorateur qui vise à récupérer la diachronie d'une langue un point de vue interne à la langue même ; tout particulièrement un point de vue interne au lexique, ce creuset du «caractère » de chaque langue, qui réunit en soi la forme phonique et la forme des idées et des choses, en forgeant ainsi sa propre « forme interne ».

\footnotetext{
${ }^{4}$ L'hypercorrection observée chez les enfants au cours de l'apprentissage de leur langue maternelle est un témoignage éloquent du fait que la notation des régularités (et de leurs anomalies) de la part des grammairiens ne vient, pour ainsi dire, qu'après coup. Cf. SGALL (2002: 309).

${ }^{5}$ Cf. Humboldt (2009).
} 
«Il est impossible de considérer le répertoire lexical d'une langue comme une somme simplement donnée et achevée. [...] Nulle mémoire humaine ne pourrait y suffire, si l'âme ne portait en elle, par une sorte d'instinct, la clef qui déverrouille l'instauration des mots eux-mêmes $»{ }^{6}$

Un tout autre effet de compréhension découle de cette façon de procéder, si on la compare avec l'autre, si répandue et d'une utilité pratique indiscutable mais tellement extrinsèque qu'elle frise l'inintelligibilité : l'ordre alphabétique.

\section{Trois niveaux d'observation: le système et sa forme interne, l'énoncé et la structure syntagmatique, l'énonciation et son origo}

Partons d'une langue et de son dictionnaire, ordonné alphabétiquement. Considérons maintenant la ville et son indicateur des rues, lui aussi organisé de la même manière. "User friendly» tous les deux, admettons-le, mais bien peu instructifs à propos de l'organisation interne des rues et des places, des ruelles et des boulevards, du centre et de la périphérie; et parallèlement, quant à l'organisation des termes primitifs, dérivés et composés, des mots pleins et des soi-disant mots vides, des co-radicaux, et ainsi de suite.

La British Library a organisé il y a quelque temps une exposition sur l'histoire - ou, mieux, sur la vie - de la ville de Londres («London, A life in maps $»^{7}$ ), où l'évolution de la ville était reconstituée à partir de ses plans.

De la même manière, il ne nous est pas impossible de récupérer des états de la langue antérieurs ou synchroniques par rapport à nous. Nous pouvons fonder notre connaissance sur la documentation métalinguistique à notre disposition - les grammaires (grammaires historiques), les dictionnaires (dictionnaires étymologiques) etc., les théories explicatives -; en plus de cela, nous pouvons avoir recours aux textes disponibles, conservés par l'écriture ou par les enregistrements sonores ou audiovisuels. Si toutefois nous constatons qu'il nous manque une prise directe sur le brouhaha dans les places ou sur les chuchotements dans les chambres les plus secrètes, nous serons obligés d'admettre qu'il existe un décalage entre la représentation, la reconstruction d'un côté et la vie (linguistique) vécue de l'autre. Un décalage, cela n'est pas un défaut, bien au contraire! Quel étranger errant, quel explorateur curieux ne se réjouiraient-ils pas d'un plan retrouvé, d'une indication mise à leur disposition?

Que de fois, un locuteur natif n'a pas su expliquer à celui qui l'interrogeait les raisons du choix d'une certaine tournure plutôt que d'une autre, encore qu'il ne fût pas question d'erreur?

Le savoir conscient est certainement une valeur. Toutefois, souvent, explorés, explorateurs et géographes sont incapables de découvrir à quel point il est beau de se fréquenter.

Puisque nous sommes persuadés qu'il vaut la peine de se fréquenter, nous allons maintenant essayer d'élargir le pouvoir suggestif qui découle de l'idée d'une corrélation entre centre et périphérie.

\footnotetext{
${ }^{6}$ Humboldt ([1836] $\left.1974: 249\right)$.

${ }^{7}$ http://www.bl.uk/onlinegallery/ecards/lim/ecards.html
} 
Nous allons ainsi essayer de traiter les systèmes linguistiques non seulement comme des entités historico-culturelles habitées de génération en génération (urbes, poleis), mais aussi d'en étudier les centres habités (tecta), d'en observer le va-et-vient des passants (cives) le long des boulevards, sur les places, les ruelles et l'enceinte des fortifications.

En laissant de côté la métaphore, encore une fois, nous essaierons de capturer la force descriptive, et, si c'est le cas, explicative, de l'idée-clé dont nous nous proposons de passer au crible l'efficacité non seulement sur l'un des niveaux, celui des systèmes linguistiques, mais également sur trois plans: les systèmes justement, mais aussi les énoncés, les énonciations. Il s'agit d'un parcours non seulement à reculons, des effets aux causes, mais aussi - et de façon complémentaire - en spirale, des potentialités communes partagées aux actualisations personnelles.

Je ne ferai qu'indiquer trois perspectives, à propos desquelles je citerai quelques pages éclairantes de maîtres se situant dans le domaine de nos études. Les perspectives qui me paraissent à la fois précieuses et prometteuses sont les suivantes :

- l'organisation interne, les dynamiques de transformation (les axes de développement) des systèmes ;

- les frontières, en évolution, des systèmes et, en même temps, leur configuration stable en tant que totalité ${ }^{8}$;

- les «pleins » et les «vides» de l'aire urbaine/linguistique; les voies de circulation; le rapport entre le tout et ses parties, entre la périphérie et le centre.

Je ne m'attarderai pas sur le premier niveau, qui fait l'objet d'un bon nombre de contributions ; c'est d'ailleurs celui qui m'appartient le moins. ${ }^{9}$

Quant au deuxième niveau, celui des agrégats qui peuplent les discours, les conversations, les textes, celui des centres habités dans lesquels s'ordonnent les édifices et les quartiers, des banlieues aux arrondissements du centre, je me bornerai à souligner tout simplement un avantage offert par l'idée de centre et de périphérie : la possibilité de saisir dans le prédicat l'élément qui assure la relation entre les syntagmes, ${ }^{10}$ et l'opportunité de hiérarchiser ces derniers afin de distinguer les arguments et les déterminants ultérieurs.

Une ville dont on visite un quartier après l'autre, un pâté de maisons après l'autre, ne sera pas vraiment explorée intégralement tant que la porte de quelques

\footnotetext{
${ }^{8}$ Langues comme systèmes ouverts mais aussi comme systèmes défendus et inaccessibles. On a opposé la ville selon Napoléon (fortifiée) à la ville selon l'utopie de Goethe (ouverte) : cf. FARA (2006:3-37).

${ }^{9}$ Néanmoins, je tiens à remercier Anne-Marie Chabrolle-Cerretini de m'avoir sollicitée à expliciter «ce que je vois » au centre d'une langue. Au plan lexical, je vois les mots radicaux ou primitifs avec leur morphologie (les dérivés et les composés étant plus périphériques), les mots fonctionnels et les déictiques. Par ailleurs, l'idiolecte de chaque locuteur comporte un système spécifique de noms propres (au moins de personnes et de lieux) : par conséquent le système de noms propres est dans chaque langue un système pluricentrique bien plus que monocentrique.

${ }^{10}$ La prédication est l'acte syntagmatique fondamental (CERCLE LinguistiQue De Prague, 1929: Thèse 2.b) ; (MATHESIUS 1926, in VACHEK 1983 : 60-61 ; MATHESIUS 1929, in VACHEK 1983 : 133); MATHESIUS 1936, in VACHEK 1966 : 317). Cf. RAYNAUd (2008).
} 
demeures ne s'ouvrira pas au visiteur, tant que le visiteur attentif ne pourra pas apercevoir le monde tel qu'on peut le voir par l'une ou l'autre de ses fenêtres. Voilà pourquoi il est nécessaire de dépasser le seuil des énoncés pour accéder au plan des énonciations, pour tenter de voir le monde du point de vue de celui qui l'habite par-ci, par-là. La ville est toujours la même, mais les systèmes de repère changent. «Je » et «tu » alternent, «ici » et «là », l'heure, l'avant et l'après se réactualisent sans cesse à partir de cette origine égocentrique ${ }^{11}$ qui est fondée par l'acte communicatif même. On entrevoit peut-être la raison de l'existence de demeures plus ou moins heureuses (au sens d'Austin).

$\mathrm{La}$ ville se prend donc à palpiter de sa propre vie, et si le glas alterne au carillonnement joyeux, c'est parce que tout ne demeure pas figé dans un éternel retour. ${ }^{12}$ Elle peut encore être assiégée ou traverser des âges d'épanouissement extraordinaire, attirer vers elle des individus ou des peuples entiers, ou se retrouver abandonnée à cause de flux migratoires plus ou moins temporaires. Avec l'aide des explorateurs et des géographes, nous pouvons la parcourir moins distraitement, nous pouvons y vivre ou lui demander l'hospitalité avec une conscience, une affection différentes. Même sans l'aide d'un plan ou d'instructions spéciales, il peut nous arriver de découvrir la présence «éphémère » d'une fleur ou d'attendre l'arrivée d'un petit prince. ${ }^{13}$

\section{Aristote, Humboldt, Wittgenstein et la tradition pragoise : sur une même longueur d'onde?}

Est-il nécessaire d'évoquer les «affinités électives » goethéennes pour justifier le rappel de divers auteurs, éloignés dans le temps, dans l'espace et éminemment originaux les uns par rapport aux autres et qui toutefois ont l'air de se laisser associer comme de formidables interlocuteurs pour assurer la triple tâche que nous venons d'esquisser?

Faisons plutôt un pas en arrière et interrogeons-nous : comment se fait-il que ce soit justement la ville qui émerge comme terme de comparaison là où la langue et les locuteurs sont convoqués en tant que deuxième terme de l'analogie ? S'agitil d'un rapprochement arbitraire et par là particulièrement discutable, ou bien, y at-il un pont qui relie les deux bords, frôlés par le même flux de pensées ?

Je laisse bien volontiers la parole à Aristote: «La communauté née de plusieurs villages est la cité, parfaite, atteignant désormais, pour ainsi dire, le niveau de l'autarcie complète : se formant pour permettre de vivre, elle existe pour permettre de vivre bien. C'est pourquoi toute cité existe par nature [...] D'après ces considérations, il est évident que la cité est une réalité naturelle et que l'homme est par nature un être destiné à vivre en cité (animal politique); celui qui est sans cité est, par nature et non par hasard, un être ou dégradé ou supérieur à

\footnotetext{
${ }^{11}$ C.-à.-d. chaque sujet parlant, l'origo du cadre énonciatif : ego-hic-nunc (BÜHLER 1934).

${ }^{12}$ Pour une réflexion sur l'opportunité de repenser, de temps à l'autre, l'idée de ville par rapport à son développement historique, voir AMIN, THRIFT (2001) ; HANNERZ (1990).

${ }^{13}$ Je fais allusion à la possibilité que le terreau des ressources linguistiques produise des fleurs différentes, spontanées ou cultivées; non seulement les «belles lettres » mais aussi une variété de messages authentiques : des déclarations d'amour ou d'engagement civil, des traits de génie, des saillies inattendues, des manifestations de pardon....
} 
Du centre à la périphérie : structuration des langues en diachronie, ou la forme interne des langues et ses axes de développement

l'homme [...]. Ainsi la raison est évidente pour laquelle l'homme est un être civique plus que tous autres, abeilles ou animaux grégaires. Comme nous le disons, en effet, la nature ne fait rien en vain; or seul d'entre les animaux l'homme a la parole (logon dé monon anthropos echei ton zoon). Sans doute les sons de la voix (phoné) expriment-ils la douleur et le plaisir ; aussi la trouve-t-on chez les animaux en général; leur nature leur permet seulement de ressentir la douleur et le plaisir et de se les manifester entre eux. Mais la parole (logos), elle, est faite pour exprimer l'utile et le nuisible et par suite aussi le juste et l'injuste. Tel est, en effet, le caractère distinctif de l'homme en face de tous les autres animaux : seul il perçoit le bien et le mal, le juste et l'injuste, et les autres valeurs ; or c'est la possession commune de ces valeurs qui fait la famille et la cité. » ${ }^{14}$

J'en viens maintenant au premier des trois moments de notre visite de la ville, à ses remparts ou à ses marges, à ses vestiges et à ses formes, à ses axes routiers, au cœur de sa fondation.

C'est Humboldt qui nous conduit à l'intérieur du noyau originaire, ${ }^{15} \mathrm{c}^{\prime}$ est Marty qui nous montre l'esquisse d'ensemble, ${ }^{16}$ c'est Wittgenstein qui nous indique les fondations de nouveaux quartiers, ${ }^{17}$ c'est Jakobson qui nous amène à reconnaître la persistance d'un tout dans la variation de ses parties, ${ }^{18} \mathrm{~d}$ 'une ville à l'autre, d'une époque à l'autre.

\footnotetext{
${ }^{14}$ ARISTOTE (1960 : 14-15 [1253a 7-20]).

15 «L'essence de la cohésion phonétique des mots repose sur l'existence d'un nombre restreint d'éléments phonétiques radicaux, constituant le support du répertoire lexical et pouvant recevoir des adjonctions et des variations qui ouvrent des possibilités de déterminations toujours plus fines et plus complexes aux concepts » (HumBoLDT ([1836] $1974: 251$ ).

${ }^{16}$ «Aucune langue n'exprime explicitement tout ce que nous souhaitons communiquer, chacune ressemble plus ou moins à un sténogramme ou à un croquis. » (MARTY $1908: 145$ ).

17 «...demande-toi si notre langage est complet ; - s'il l'était avant que le symbolisme chimique et la notation infinitésimale lui aient été incorporés; car ce sont, pour ainsi dire, des faubourgs de notre langage. (Et à partir de combien de maisons ou de rues une ville devient-elle une ville ?) On peut considérer notre langage comme une ville ancienne, comme un labyrinthe fait de ruelles et de petites places, de maisons anciennes et de maisons neuves, et d'autres que l'on a agrandies à différentes époques, le tout environné d'une multitude de nouveaux faubourgs avec leurs rues tracées de façon rectiligne et régulière, et bordées de maisons uniformes. » (WITTGENSTEIN [1953] 2007 : I, 18).

18 «All cognitive experience and its classification is conveyable in any existing language. Whenever there is a deficiency, terminology can be qualified and amplified by loanwords or loan-translations, by neologisms or semantic shifts, and, finally, by circumlocutions. [...] No lack of grammatical devices in the language translated into makes impossible a literal translation of the entire conceptual information contained in the original. [...] If some grammatical category is absent in a given language, its meaning may be translated into this language by lexical means. Dual forms like Old Russian brata are translated with the helps of the numeral : 'two brothers'. It is more difficult to remain faithful to the original when we translate into a language provided with a certain grammatical category from a language lacking such a category. [...] As Boas neatly observed, the grammatical pattern of a language (as opposed to its lexical stock) determines those aspects of each experience that must be expressed in the given language [...]. Languages differ essentially in what they must convey and not in what they can convey » (JAKOBSON ([1959] $1971: 263-264)$. Cf. aussi JAKOBSON ([1963] 1971: 280-281): "The fact that all this entities, from the discourse to its ultimate components (distinctive features), have quite different statuses in respect to the verbal code and present diverse degrees of relative dependence does not justify the attempts to exclude some of these units from the realistic and comprehensive portrayal of language as it actually is - a multistored hierarchy of wholes and parts ».
} 
Ce n'est que dans un souci de synthèse que je renonce ici à des pages d'auteurs pragois de la première, ${ }^{19}$ deuxième ${ }^{20}$ ou troisième génération, ${ }^{21}$ ou tchèques ${ }^{22}$ portant sur les notions de «centre » et «périphérie » dans les systèmes linguistiques.

Si ensuite nous considérons avec Cicéron l'urbs en tant que conjunctio tectorum, comment pouvons-nous oublier la tectogrammaire de l'école de Prague, au sommet des relations morphonologico-lexico-syntaxiques, esquissées par les grammaires dépendencielles ? Comment ne pas admettre dans le tableau urbain les forêts d'arbres syntaxique dont les branches et les feuilles se départent de chaque prédicat et dessinent chaque énoncé ? ${ }^{23}$

Le fait de pouvoir pousser notre regard des arbres aux champs tout en restant dans la ville ${ }^{24}$ nous fait respirer à pleins poumons.

Nous sommes redevables à Bühler pour le rapprochement qu'il opère entre le champ symbolique et le champ d'indication, et pour l'individuation de centres déictiques mobiles aussi nombreux que le sont les locuteurs et leurs prises de parole. Par ailleurs, le rôle des locuteurs, les effets de leur acte d'énonciation n'échappent pas non plus à Humboldt ${ }^{25}$, qui tire son inspiration de phénomènes naturels pour illustrer l'action réciproque que la langue exerce sur les locuteurs et ces derniers sur elle. C'est l'exemple des cercles concentriques qui se propagent sur l'eau, ou des cristaux qui se forment petit à petit.

«Nul ne donne au mot exactement la même valeur qu'autrui; toute différence, si faible soit-elle, provoque, comme les cercles que fait une pierre dans l'eau, des remous qui se répercutent à travers toute la surface de la langue. ${ }^{26}$

«La genèse de la langue ressemble, pour prendre un terme de comparaison dans la nature physique, au processus de cristallisation. L'instauration se produit peu à peu, mais selon une loi. $\gg^{27}$

\section{Métaphores constitutives et implications métathéoriques, épistémologiques, méthodologiques au début du XXI ${ }^{\text {ème }}$ siècle}

Jusqu'où est-il permis de se laisser transporter par les similitudes, ou de s'arrêter aux analogies ? Est-il admissible de se rendre à la force de persuasion des rapprochements ingénieux, à la fascination - pour autant qu'elle soit perçue - des suggestions qui nous soulagent du pénible travail sur les données et sur leur élaboration spécifique?

Je trouve donc on ne peut plus appropriée la correction de route ou, mieux encore, l'indication plus nette de la trajectoire à suivre qui découle de la notion de « métaphore constitutive»: «There exists an important class of metaphors which play a role in the development and articulation of theories in relatively mature

\footnotetext{
${ }^{19} \mathrm{Cf}$. MATHESIUS et son réalisme.

${ }^{20}$ DANEŠ (1966) ; LEŠKA (1966) ; NEUSTUPNÝ (1966) ; VACHEK (1966) ; FILIPEC (1966).

${ }^{21}$ SGALl (2002); HajIČOVÁ (2006).

${ }^{22}$ RADIMSKÝ (2007).

${ }^{23}$ HAJIČOVÁ (2002).

${ }^{24}$ HAJIČOVÁ (2006 : 465).

${ }^{25}$ HumboldT ([1820] ; [1836]).

${ }^{26}$ Humboldt ([1836] $1974: 203$ ).

${ }^{27}$ Humboldt ([1836] $\left.1974: 318\right)$.
} 
sciences. Their function is a sort of catachresis - that is, they are used to introduce theoretical terminology where none previously existed. [...] There are [...] cases in which complete explication is impossible, but far from being indications of the imprecision of metaphorical language in science, such cases reflect the necessity of obtaining a precise fit between scientific language and a messy and complex world. [...] More precisely, what I shall argue is that the use of metaphor is one of many devices available to the scientific community to accomplish the task of accommodation of language to the causal structure of the world. ${ }^{28}$

La délicatesse de notre étude de cas est soulignée par le fait que notre tâche est celle de modeler le langage sur la structure effective du langage.

Nous ne pouvons à ce point que faire allusion aux nombreuses disciplines, diachroniques ou synchroniques, descriptives ou normatives, théoriques et appliquées auxquelles on fait appel quand il s'agit d'explorer, de passer au peigne fin nos villes, de déterminer de nouvelles constructions, de restructurer les anciennes.

Comme il n'est pas banal d'approuver un nouveau plan d'urbanisme, ou plus simplement de projeter un nouveau quartier ou de déplacer certains services sur le plan d'urbanisation, de la même manière, cela ne va pas de soi de décider à quelle école faire confiance, à quel auteur se référer afin d'être sûr de résoudre au mieux la tâche à affronter, sans céder à des pressions ou à des intimidations ou, tout simplement, sans se laisser entraîner par la force de l'habitude ou les conformismes à la mode. Comme pour tout choix, les marges de liberté et de conscience peuvent varier. Chacun de nous a fait et continue à faire ses propres choix: de sélection des données sur lesquelles intervenir, de méthode, d'organisation de sa discipline, d'adhésion à une approche théorique déterminée.

L'occasion est très rare de pouvoir évaluer l'efficacité d'une idée, sa fécondité théorique et appliquée et de pouvoir saisir ses retombées sur ses propres travaux en cours. À ceux qui nous en ont offert l'opportunité et à l'école qu'ils représentent, j'adresse mon remerciement le plus cordial et chaleureux.

\section{RÉFÉRENCES BIBLIOGRAPHIQUES}

Amin, Ash, Thrift Nigel (2001), Cities. Reimagining the Urban, Cambridge, Polity Press.

Aristote (1960), Politique. Livres I et II. Texte établi et traduit par Jean Aubonnet, Paris, Les Belles Lettres.

BOYD, Richard (1994, $2^{\mathrm{e}}$ éd.), Metaphor and theory change : What is "metaphor" a metaphor for? In: A. Norton (éd.), Metaphor and Thought, Cambridge, Cambridge UP.

BüHLER, Karl (1934, $1^{\mathrm{e}}$ éd.), Sprachtheorie. Die Darstellungsfunktion der Sprache, Jena, Fische ; (2009), traduit en français par Didier Samain Theorie du langage, Marseille, Agone.

28 (BOYD, 1994, $2^{\mathrm{e}}$ éd. : 482-483). 
BüHLER, Karl (1936), Das Strukturmodell der Sprache, in : Travaux du Cercle Linguistique de Prague 6, p. 3-12 ; (2006) traduit de l'allemand par M. Paola Tenchini, Il Modello strutturale del linguaggio, in : Savina Raynaud (éd.), $T u$, io, qui, ora. Quale semantica per gli indicali? Milano, Guerini, p. 174-182.

Cercle Linguistique de Prague (1929): Thèses présentées au Premier Congrès des philologues slaves, in: Travaux du Cercle linguistique de Prague 1: Mélanges linguistiques dédiés au Premier Congrès des philologues slaves, Prague, Jednota československých matematiků a fysiků, p. 5-29.

DANEŠ František (1966), The relation of centre and periphery as a language universal, in: Travaux Linguistiques de Prague 2. Les problèmes du centre et de la périphérie du système de la langue, Prague, Éditions de l'Académie tchécoslovaque des sciences, p. 9-21.

FARA, Amelio (2006), Napoleone architetto nelle città della guerra in Italia, Firenze, Olschki.

FILIPEC, Josef (1966), Probleme des Sprachzentrums und der Sprachperipherie im System des Wortschatzes, in: Travaux Linguistiques de Prague 2. Les problèmes du centre et de la périphérie du système de la langue, Prague, Éditions de l'Académie tchécoslovaque des sciences, p. 257-274.

FORCELLINI, Egidii (1805), Totius latinitatis lexicon / consilio et cura Jacobi Facciolati; opera et studio Ægidii Forcellini lucubratum Patavii, Typis Seminarii, 4 voll.

HAJIČOVÁ, Eva (2002), Theoretical description of language as a basis of corpus annotation: The case of Prague Dependency Treebank, in E. Hajičová, P. Sgall, J. Hana \& T. Hoskovec (eds.), Travaux du Cercle linguistique de Prague, n. s. / Prague Linguistic Circle Papers 4. Amsterdam-Philadelphia, John Benjamins, p. 111-127.

HAJIČOVÁ, Eva (2006), Old Linguists Never Die, They Only Get Obligatorily Deleted, Computational Linguistics 32 (4), p. 457-469.

HANNERZ, Ulf (1990), Exploring the cities. Inquiries Toward an Urban Anthropology, New York, Columbia University Press.

HUMBOLDT, Wilhelm von ([1820] 1963), Über das vergleichende Sprachstudium in Beziehung auf die verschiedenen Epochen der Sprachentwicklung, in Id., Werke in fünf Bänden, hrsg. v. Andreas Flitner und Klaus Giel, Stuttgart; (1974), traduit en français par Pierre Caussat, Introduction à l'œuvre sur le kawi et autres essais, Paris, Éditions du Seuil, p. 71-96.

HUMBOLDT, Wilhelm von ([1836] 1998), Über die Verschiedenheit des menschlichen Sprachbaues und ihren Einflu $\beta$ auf die geistige Entwicklung des Menschengeschlechtes. Paderborn, München, Wien, Zürich, F. Schöningh ; (1974), traduit en français par Pierre Caussat, Introduction à l'ouvre sur le kawi et autres essais, Paris, Éditions du Seuil, p. 133-420.

HUMBOLDT, Wilhelm von (2009), Südamerikanische Grammatiken III, hrsg von Manfred Ringmacher, Ute Tintemann, Paderborn, Ferdinand Schöningh.

JAKOBSON, Roman (1959), On linguistic aspects of translation, in: On Translation, Cambridge, Mass., Harvard University Press ; in: (1971), Selected Writings II. Word and Language, Berlin-New York, Mouton De Gruyter, p. 260-266. 
Du centre à la périphérie : structuration des langues en diachronie, ou la forme interne des langues et ses axes de développement

JAKOBSON, Roman (1963), Parts and wholes in language, in Parts and Wholes, ed. by D. Lerner, New York-London, Free Press of Glencoe ; in (1971), Selected Writings II. Word and Language, Berlin-New York, Mouton De Gruyter, p. 280-284.

LEŠKA, Oldřich (1966), «Le centre » et « la périphérie » des différents niveaux de la structure linguistique, in: Travaux Linguistiques de Prague 2. Les problèmes du centre et de la périphérie du système de la langue, Prague, Éditions de l'Académie tchécoslovaque des sciences, p. 53-57.

MARTY, Anton (1908), Untersuchungen zur Grundlegung der allgemeinen Grammatik und Sprachphilosophie, Halle a. S., Niemeyer; reprint (1976) Hildesheim-New York, Olms.

MATHESIUS, Vilém (1926), New Currents and Tendencies in Linguistic Research, in : MNHMA : Festschrift for Josef Zubatý, Praha, p. 188-203 ; (1983) reprint in : Josef Vachek (éd.), Praguiana: Some Basic and Less Known Aspects of the Prague Linguistic School. An Anthology of Prague School Papers, Prague, Academia, p. 45-63.

MATHESIUS, Vilém (1929), Funkční lingvistika, in: Sborník přednášek proslovených na Prvém sjezdu čsl. profesorü filosofie, filologie a historie v Praze 3.-7. dubna 1929, Praha, Stálý přípravný výbor sjezdový, p. 118-130 ; version anglaise in: Josef Vachek (1983, éd.), Praguiana: Some Basic and Less Known Aspects of the Prague Linguistic School. An Anthology of Prague School Papers, Prague, Academia, p. 121-142.

MATHESIUS, Vilém (1936), Deset let Pražského linguistického kroužku. Slovo a slovesnost 2: 137-145; version anglaise in: Josef Vachek (1966, éd.), The Linguistic School of Prague: An Introduction to its Theory and Practice Bloomington, Indiana University Press, p. 137-151.

NEUSTUPNÝ, Jiří V. (1966), On the analysis of linguistic vagueness, Travaux Linguistiques de Prague (TLP) 2. Les problèmes du centre et de la périphérie du système de la langue, Prague, Éditions de l'Académie tchécoslovaque des sciences, p. 39-51

RADIMSKÝ, Jan (2007), De la potentialité à la synchronie dynamique : un héritage oublié du Cercle de Prague, in: Radimský, Jan (éd.) (2007), Synchronie dynamique du système linguistique, numéro thématique de l'Écho des études romanes, vol. III / Num. 1-2, p. 7-12; version électronique : [http://www.eer.cz/tab1.html].

RAYNAUD, Savina (2008), The basic syntagmatic act is predication, Slovo a slovesnost, 69, p. 49-66.

SGALl, Petr (2002), The freedom of language, Prague Linguistic Circle Papers 4, Amsterdam - Philadelphia, John Benjamins, p. 309-329.

SGALl, Petr, BöHMOVÁ, Alena (2002), The simple core and the complex periphery of natural language - a formal and a computational view, Proceedings of the 19th International Conference on Computational Linguistics (COLING 2002), San Francisco, Morgan Kaufmann Publishers, p. 925-931.

VACHEK, Josef (1966), On the integration of the peripheral elements into the system of language, in : Travaux Linguistiques de Prague 2. Les problèmes $d u$ 
centre et de la périphérie du système de la langue, Prague, Éditions de l'Académie tchécoslovaque des sciences, p. 23-37.

Wittgenstein, Ludwig (1953), Philosophical Investigations, tr. by Gertrude Elizabeth Margaret Anscombe, Oxford, Basil Blackwell ; (2007) traduit de l'allemand par Françoise Dastur, Maurice Elie, Jean-Luc Gautero, Dominique Janicaud, Elisabeth Rigal, Recherches philosophiques, Paris, Gallimard.

\section{SUMMARY}

Since it is difficult to define language, an effective method of proceeding is by deepening and strengthening the knowledge of its properties, in particular through comparisons.

This paper aims to clarify the well known property of human languages, which are characterized by having a centre and a periphery. The notions of centricity-peripherality, developed within the Prague linguistic tradition, apply to many other entities, both cultural and natural: towns first of all, but also physiological systems (the circulatory and nervous systems), and physical phenomena (the formation of crystals, the propagation of circles in the water resulting from the impact of a water droplet...).

The lines followed are twofold: i. the primacy of the linguistic activity of the community of speakers over metalinguistic considerations; ii. the identification of three layers of the metalinguistic enterprise: the system (lexicon and grammar) and the inner form of its structuring; the sentence and its syntagmatic structure; the utterance and its indexical origin.

The Praguian linguistic tradition finds itself in conversation with authors from outside its ranks, from Aristotle to Wittgenstein, through Humboldt and Marty.

If science needs metaphors and can't do without them, constitutive metaphors have to be preferred. The metaphor of centre and periphery proves to be one of these. 